$\mathrm{Xu}, \mathbf{N}$.

\title{
Spacio-Temporal Development of the Rural Natural Landscape in the Beijing-Tianjin Region
}

\author{
Ninghan XU, Tianjin University, China
}

\begin{abstract}
Creating a good natural rural landscape is thus an inherent part of building rural residences in which people and nature live in consonance. China endeavors to build an eco-friendly society, under the background of which rural areas are given a high priority in the systematic protection and utilization of national land resources. This essay confines its investigation to the cities of Beijing and Tianjin. It acquires data on the rural natural landscape by interpreting images generated by remote sensing and analyzing spatial layouts of rural areas. Research shows that, in terms of the development of material forms, rural natural landscapes in the Beijing-Tianjin region are mostly either agricultural lands or woodlands. In addition, they also possess four spatial characteristics, namely: have evident spatial differences and four spatial characteristics includes scale, distribution, network and shape.During rapid urbanization, the development in elements of rural natural landscapes in the Beijing-Tianjin region can be roughly summarized as the transformation of agricultural lands into construction lands. Construction lands expand to other surrounding areas, gradually consuming the rural natural landscape and appropriating its space. As a result, the area of the rural natural landscape decreases, its fragmentation worsens, and the heterogeneous shape has continued to fluctuate.
\end{abstract}

\section{Keywords}

Rural Natural Landscape, Rural Landscape, Landscape Pattern, Spacio-Temporal Development, Urbanization, Beijing-Tianjin Region

\section{Introduction}

\subsection{Subheading}

China has vast rural areas, which take up approximately $94 \%$ of the nation's total lands. Rural life is the basic relationship between people and land in various types of human settlements. Its development depends heavily on the areas' cultures, and the feelings, most remarkably nostalgia, of the people from these regions. It is an organic combination of extrinsic material forms and intrinsic cultural spirits of the area. It is therefore an important element of the environment of human settlements.

In the world of human activities, China's ongoing rapid urbanization is unique. The rapid urbanization process has led to unprecedented intensification of human activities and dramatic changes in urban and rural areas. In this context, it is of great significance to study the evolution law of rural natural landscape and explore the human-land interaction mode in rural space to construct harmonious and symbiotic human-land relationship in rural areas.

At present, there are few studies at home and abroad to show the rural landscape and summarize the 
space-time characteristics of rural natural elements from the perspective of rural natural landscape. Most of them are analyzed from the perspectives of rural architecture construction, landscape design, social culture and other artificial landscape and cultural landscape. Yuan Qing, a Chinese scholar, has carried out studies on some natural landscape features in the cold northeast plain from the perspectives of rural landscape toughness ${ }^{[1]}$ and the construction of complex landscape features system between city and township ${ }^{[2]}$. However, there are still few studies on non-cold areas and quantitative analysis of rural natural landscape features. Therefore, the study of the spatial and temporal evolution of rural natural landscape features in Beijing and Tianjin can supplement the blank of rural natural landscape features in plain areas of China.

At present, most researches on rural natural landscape are based on rural landscape, and rural landscape is a subcategory of rural and urban landscape. Urban and rural landscape is made up of the natural environment, urban and rural amorous feelings of historical tradition, modern, comprehensive constitute the image such as spiritual culture, not only contains the visible space landscape, architectural form, color, pattern of landscape and greening, etc.), also contains the temperament of the verve of the urban and rural areas, places of public spirit, customs, culture and science and culture, is the main embodiment of urban and rural characteristics. Urban and rural landscape is an important part of the overall image of urban and rural areas, reflecting the historical, cultural and social development of a particular region.

In the field of urban and rural planning, studies on urban and rural landscape are generally divided into urban landscape and rural landscape. In recent years, there are more studies on urban landscape and less on rural landscape. At present, the typical research object of rural landscape in China is the artificial elements of rural landscape, such as village construction, village infrastructure, landscape design and so on. Based on the investigation of 480 villages in 13 provinces of China and the investigation of villages at home and abroad in recent years, Zhang Li et al. sorted out and summarized the current crisis of Chinese rural landscape, such as the gradual loss of regional differentiation, significant urban representation, chaotic and artificial site landscape, rural environment delocalization and ecological characteristics. The village society is gradually deconstructed, and the material and intangible heritage is difficult to continue $^{[3]}$.

\section{Methodology}

\subsection{Data sources}

Remote sensing images used in this paper come from Data Center for Resources and Environment, Chinese Academy of Sciences ${ }^{[4]}$ (http://www.resdc.cn). Landsat-tm remote sensing images of Beijing and Tianjin in 1980, 1990, 2000, 2010 and 2018 were obtained as the main data Source of information, image resolution of $30 \mathrm{~m}$, time span of 38 years. Considering the instantaneous quality of remote sensing information acquisition in the study area (such as cloud measurement $<10 \%$ and other technical indicators), in terms of seasonal phase, images from early May to mid-October were selected in this paper.

In this paper, the use of population, economy, agriculture, land use and social statistical data from 1980 to 2018, the Beijing and tianjin statistical yearbook, statistical bulletin, "tianjin local Chronicles" (published in February 2013, local Chronicles compilation committee), "county", the second national land survey report, the national land change survey report and related data.

\subsection{Spatial pattern analysis based on GIS}

ArcGIS10.0 software was used to analyze the spatial pattern of five periods of remote sensing image classification data from 1980 to 2018 in Beijing and Tianjin, and generate the distribution map of rural 
natural landscape in Beijing and Tianjin, and statistical information of various landscape patches. Specific operations are as follows:

(1) Reclassification

The administrative vector maps of Beijing and Tianjin were superimposed and cropped with remote sensing images of the five phases to generate remote sensing image vector data of the study area. The reclassification tool of Spatial Analyst module is used to reclassify the patches of the classification data of remote sensing images in five phases according to cultivated land, forest land, grassland, water area, construction land and unused land and adjust the display color blocks.

(2) Partition geometry statistics

The zonal geometric statistics tool can calculate the area of individual landscape patches in a raster data set. The area, patch number, perimeter and other information of each landscape type in the five remote sensing images in Beijing and Tianjin are calculated by using the partition geometric statistics tool in Spatial Analyst module.

(3) Transfer matrix construction

The transition matrix can represent the related transition between landscape types in two time periods and reflect the information of landscape structure change, dynamic process and changing patch. The specific information includes the area, direction, patch number, patch name and other information of the transfer between different landscape types. Its expression is:

$$
S_{i j}=\left[\begin{array}{ccc}
S_{11} & \cdots & S_{1 n} \\
\vdots & \ddots & \vdots \\
S_{n 1} & \cdots & S_{n n}
\end{array}\right](1)
$$

In the formula, Sij is the state of landscape in the initial period and the final period, and $\mathrm{N}$ is the number of landscape types. Vector is the area of each type of landscape and represents the composition type of landscape structure in different time periods.

Data Management Tools/Substitute /Dissolve tool is selected in ArcGIS10.0 software to perform Data fusion for remote sensing images in each time period, and then Intersect tool is used to superposition remote sensing images. Finally, Calculate Geometry tool is used to Calculate and derive data.

\subsection{Landscape index analysis based on Fragstats}

(1) Selection of landscape index

In this paper, the analysis of rural natural landscape in Beijing and Tianjin is divided into the space-time evolution of rural natural landscape elements and the change of rural natural landscape pattern. Accordingly, the landscape index at the type level and landscape index at the landscape level are selected respectively.

On the factor dimension, CA, NP, PD, LPI, ED, AREA_MN, FRAC_MN, IJI, AI, COHESION,PLAND were selected.On the pattern dimension, NP, PD, LPI, ED, LSI, AREA_MN, CONTAG, COHESION, DIVISION, SPLIT, SHDI, SHEI were analyzed.

(2) Classification of landscape index

The landscape indices of 11 types were classified according to five categories: representation scale, fragmentation, concentration, network degree and shape. The landscape indices of the 12 landscape levels were classified by fragmentation, edge effect, connectivity, separation and heterogeneity . 
(3) Calculation of landscape index

In this paper, ArcGIS10.0 software was used to set the natural landscape classification data of five periods from 1980 to 2018 in Beijing and Tianjin area at the same resolution $\left(30 \mathrm{~m}^{*} 30 \mathrm{~m}\right)$, and Fragstats4.2 software was imported to process the data according to the selected landscape index, and the landscape index of each year type level and landscape level in Beijing and Tianjin area was obtained. This paper analyzes the evolution of rural landscape types and patterns in Beijing and Tianjin.

\section{Results and Discussion}

Based on landscape index analysis of rural natural landscape in Beijing and Tianjin in 2018 and spatial statistical analysis of land use landscape classification data in Five periods from 1980 to 2018, this chapter analyzes and summarizes the spatial differentiation and characteristics of rural natural landscape and the evolution law of natural landscape types. The spatial-temporal evolution of rural natural landscape elements in Beijing and Tianjin from 1980 to 2018 was summarized.

\subsection{Spatial differentiation of rural natural landscape}

The spatial differentiation of rural natural landscape in Beijing and Tianjin is significant, forming a natural landscape pattern of "mountain, field and sea", with four dimensions of spatial characteristics of scale, distribution, network and shape. However, the high spatial differentiation of rural natural landscape types is not balanced on the whole, leading to the simplicity of rural surrounding natural landscape types, and the rural landscape features in Beijing and Tianjin are not obvious (Figure 3.1).

The rural natural landscape in Beijing and Tianjin is mainly cultivated land landscape and woodland landscape. Woodland landscape and grassland landscape are concentrated in the northwestern mountainous area, while water landscape originates from the mountainous area, flows through the villages and farmland landscape in the central part, and gathers in the southeast to form a water system and finally enters the Bohai Sea, forming a natural landscape pattern of "mountain-field-sea".

The patches of cultivated land landscape and woodland landscape were large and abundant, the patches of grassland landscape were small and abundant, and the patches of water landscape had the largest number and density. The order of fragmentation was water landscape $>$ grassland landscape $>$ cultivated land landscape > woodland landscape, and the order of concentration was woodland landscape > cultivated land landscape $>$ water landscape $>$ grassland landscape. Water landscape patches are abundant, scattered, highly connected and networked. The shape of cultivated landscape is most complex and irregular.

However, the high spatial differentiation of rural natural landscape types in Beijing and Tianjin is not balanced on the whole, leading to the unitary nature landscape types around the countryside, and the rural landscape features in Beijing and Tianjin are not obvious. At present, the rural natural landscape in Beijing and Tianjin area is still in the relatively primitive stage, and the process of improving the quality of regional landscape has not been realized through the overall and balanced design of rural natural landscape from the regional perspective. 


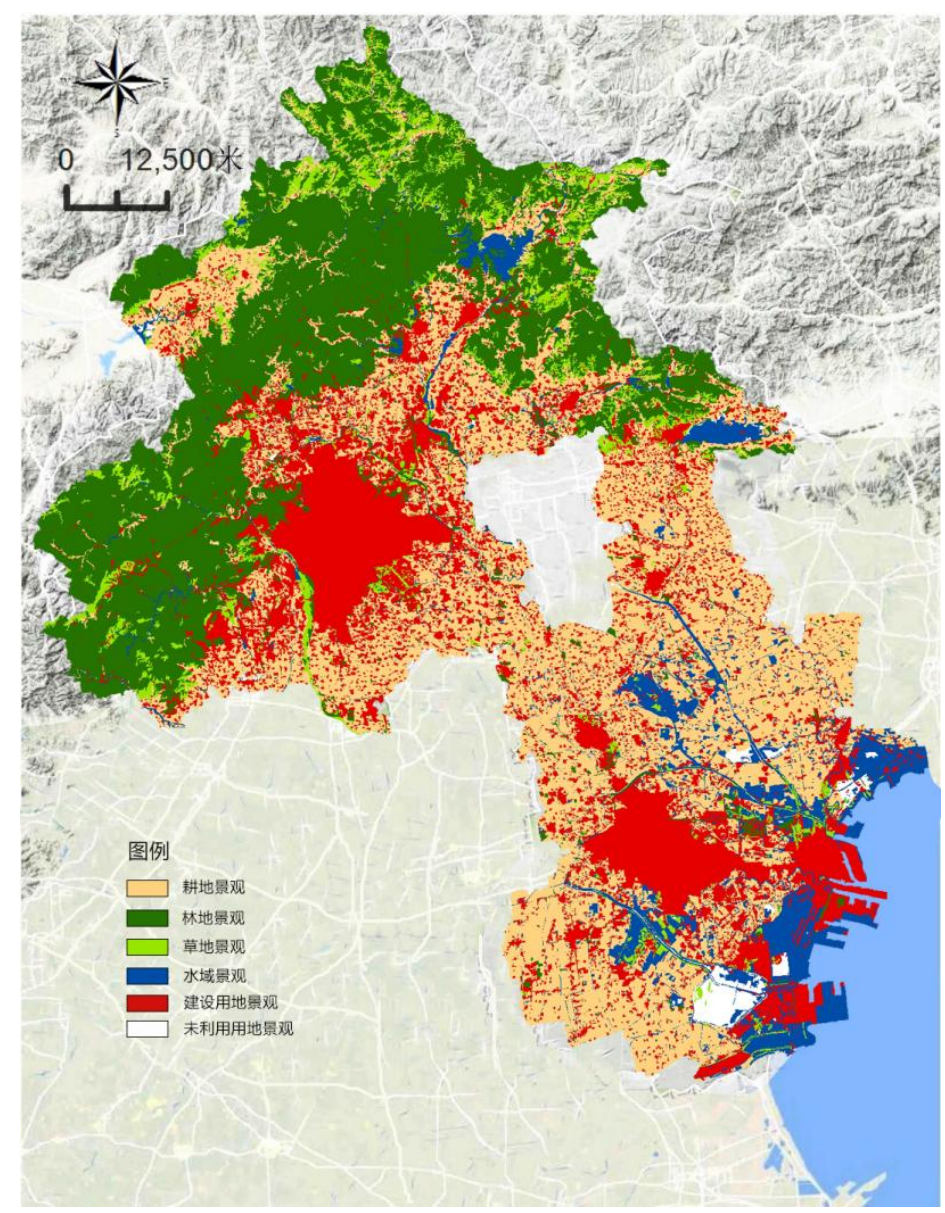

Figure 3.1 Distribution of rural natural landscape in Beijing and Tianjin in 2018. Source: Photo by author.

\subsection{Structural changes of rural natural landscape elements}

The construction land is centered on the urban center and rural residential areas, and expands to the periphery in a circular manner, while the cultivated land landscape shrinks and presents a fragmented structure.

During the past 40 years from 1980 to 2018 (Figure 3.2), with the rapid urbanization, the construction land landscape in Beijing and Tianjin maintained an expanding trend, while the arable land landscape land continued to shrink. As can be seen from the distribution map of rural landscape types in Beijing and Tianjin from 1980 to 2018, construction land is centered on urban central area and rural residential area and expands to the periphery in a circular way. The main cores are Beijing urban central area, Tianjin urban central area and Binhai New Area. Construction land kept expanding rapidly from 1980 to 2010, and slowed down after 2010. From 1990 (Figure 3.3) to 2000 (Figure 3.4), construction land expanded the most, showing a patchy distribution, and began to gather in the central city and towns, gradually forming a network form. Due to the geographical characteristics, there is a large area of cultivated land around the construction area in Beijing and Tianjin, which provides conditions for the expansion of construction land. Therefore, while the construction land is expanding in circles with three central points and scattered rural settlements as the core, the cultivated land landscape is also shrinking in circles, and the whole cultivated land landscape is segmented and tends to be fragmented.

A small amount of water landscape was eroded in the process of construction land expansion, but the overall area increased and the overall network structure was strengthened.In the process of construction land expansion, a small amount of surrounding water landscape was eroded. However, from the 
perspective of the distribution of rural natural landscape, the area of water landscape increased from 1980 to 2010 (Figure 3.5), and gradually gathered, and its network structure was strengthened.

Woodland landscape and grassland landscape are concentrated in the mountainous areas of northwest China, far from the urban center and rural settlements, and are less affected by human activities. From 1980 to 2018 (Figure 3.1), woodland landscape and grassland landscape kept their spatial characteristics, and the area and spatial structure did not change significantly.

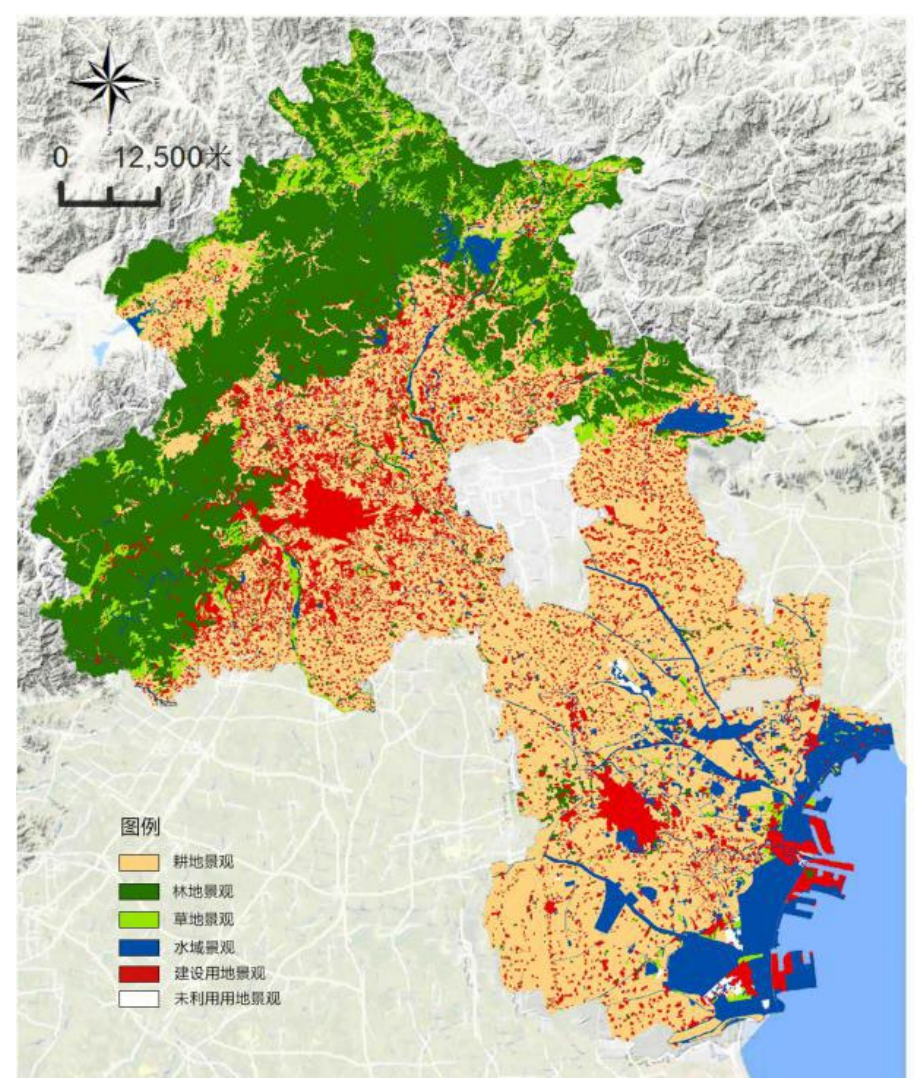

Figure 3.2 Distribution of rural natural landscape in Beijing and Tianjin in 1980. Source: Photo by author. 
$\mathrm{Xu}, \mathbf{N}$.

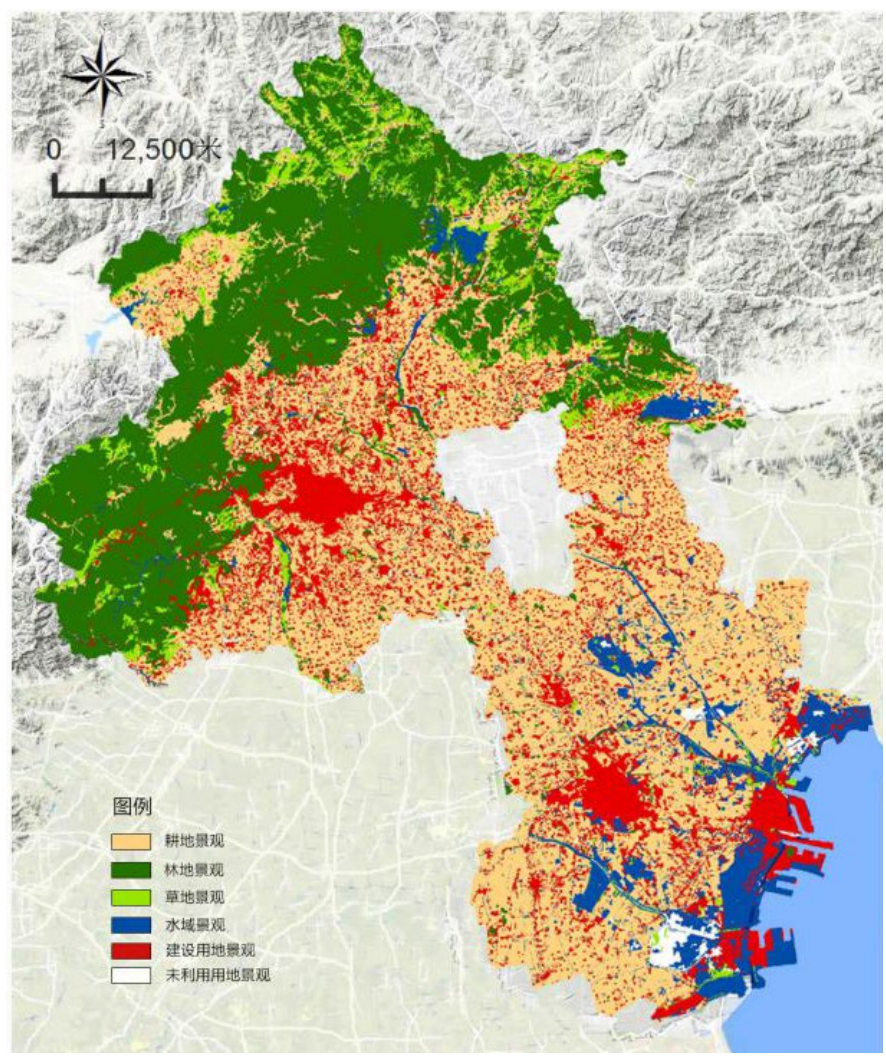

Figure 3.3 Distribution of rural natural landscape in Beijing and Tianjin in 1990. Source: Photo by author.

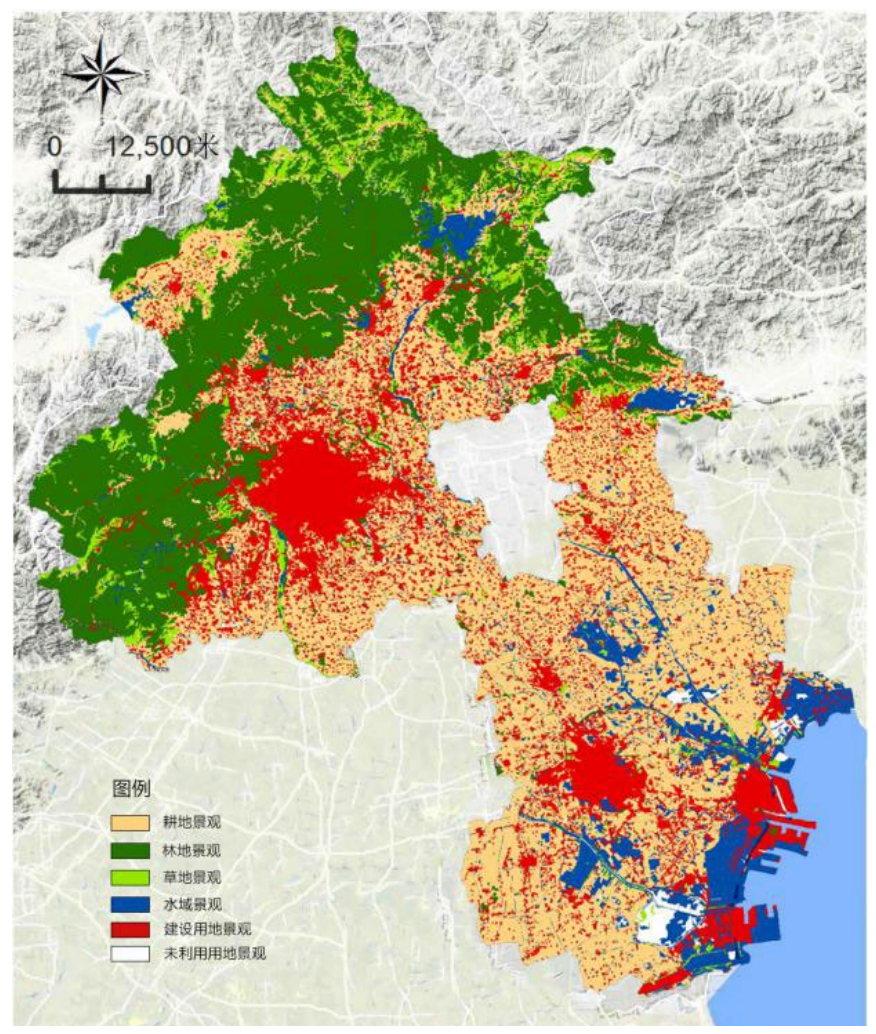

Figure 3.4 Distribution of rural natural landscape in Beijing and Tianjin in 2000. Source: Photo by author. 


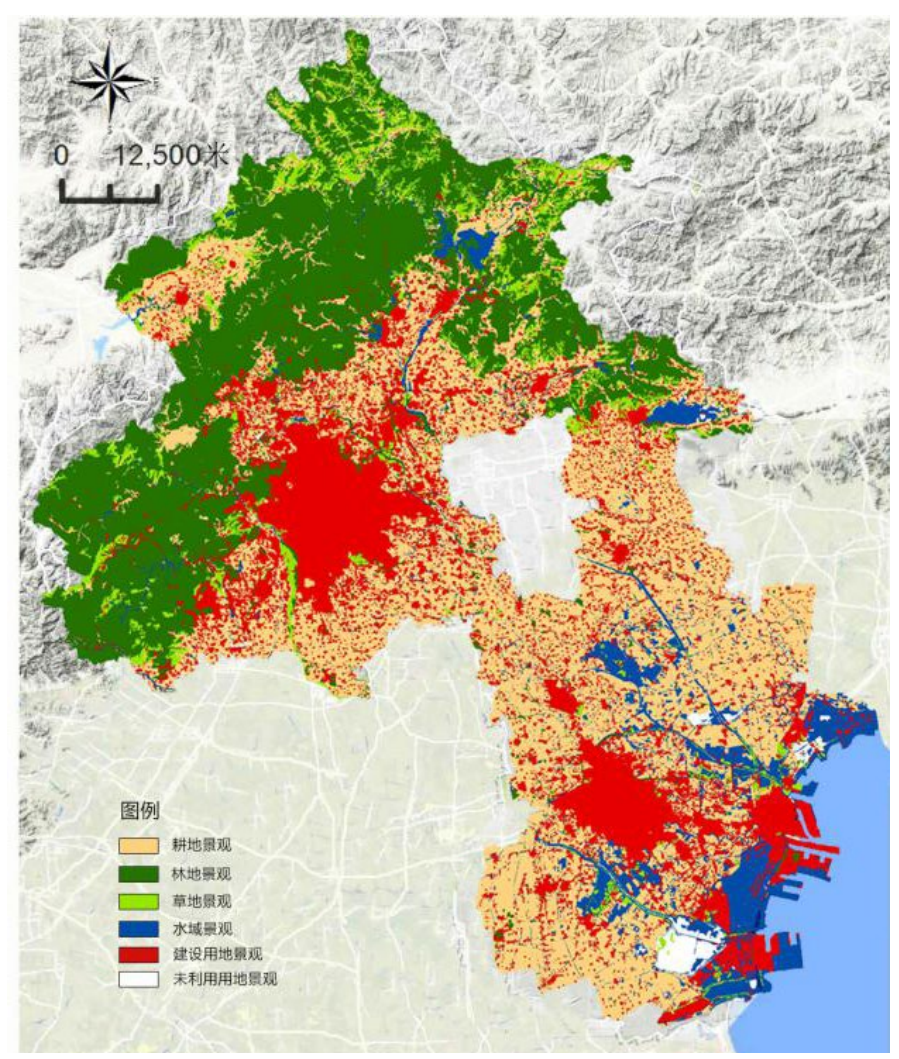

Figure 3.5 Distribution of rural natural landscape in Beijing and Tianjin in 2010. Source: Photo by author.

\subsection{Transfer direction of rural natural landscape elements}

According to the data analysis, from 1980 to 1990, arable land landscape and water landscape shifted to non-natural landscape in a large range, while woodland landscape and grassland landscape shifted to nonnatural landscape in a small range (figure 3.6). From 1990 to 2000, the trend of shifting from cultivated land landscape and water landscape to non-natural landscape slowed down, while the trend of shifting from woodland landscape to grassland landscape remained stable (figure 3.7). From 2000 to 2010, the number of natural landscape transferred to non-natural landscape decreased significantly (figure 3.8). From 2010 to 2018, the area of farmland landscape converted to non-natural landscape continued to decline, with a greater decline, showing an obvious trend of continuous decline (figure 3.9).

In the process of rapid urbanization, the change of rural natural landscape elements in Beijing and Tianjin is mainly the process of shifting from cultivated land landscape to construction land landscape, and the mutual transfer of rural natural landscape elements is small.

The landscape of construction land in Beijing and Tianjin increased by $3 \%$ per decade, while the landscape of arable land decreased by $3 \%$ per decade. The types of rural natural landscape elements in Beijing and Tianjin are not rich enough and mainly cultivated land landscape, while the conversion of cultivated land landscape into construction land landscape is low cost and easy to operate. Meanwhile, the expansion of metropolis needs to maintain a certain agglomeration effect, which causes the continuous transfer of cultivated land landscape to construction land landscape. 
$\mathbf{X}, \mathbf{N}$.

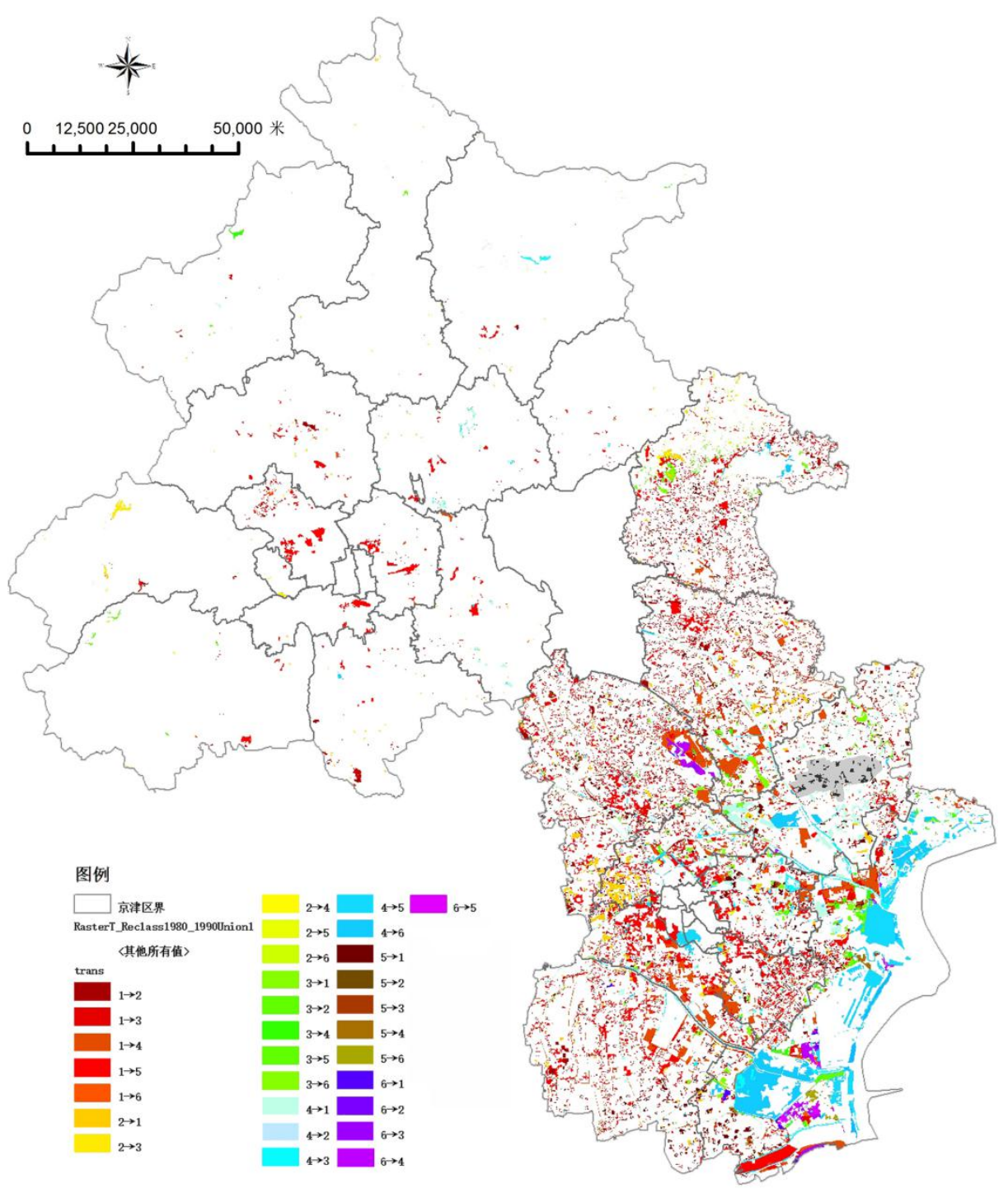

Figure 3.6 Distribution of landscape type transfer in 1980-1990(In the legend, "1", "2", "3", "4", "5" and "6" respectively represent cultivated land landscape, woodland landscape, grassland landscape, water landscape, construction land landscape and unused land landscape.)Source: Photo by author. 
$\mathbf{X}, \mathbf{N}$.

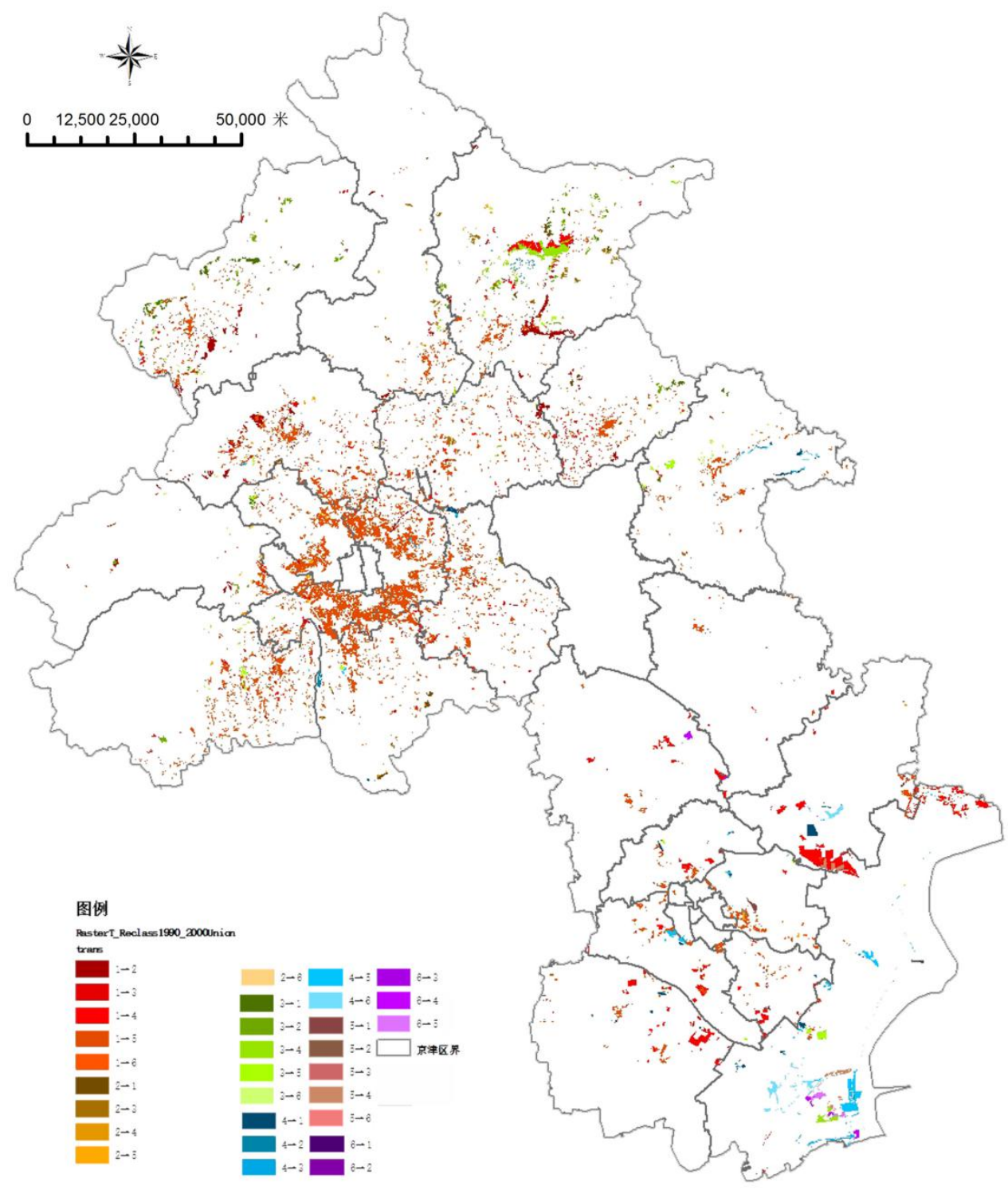

Figure 3.7 Distribution of landscape type transfer in 1990-2000(In the legend, "1", "2", "3", "4", "5" and "6" respectively represent cultivated land landscape, woodland landscape, grassland landscape, water landscape, construction land landscape and unused land landscape.)Source: Photo by author. 
Xu, N.

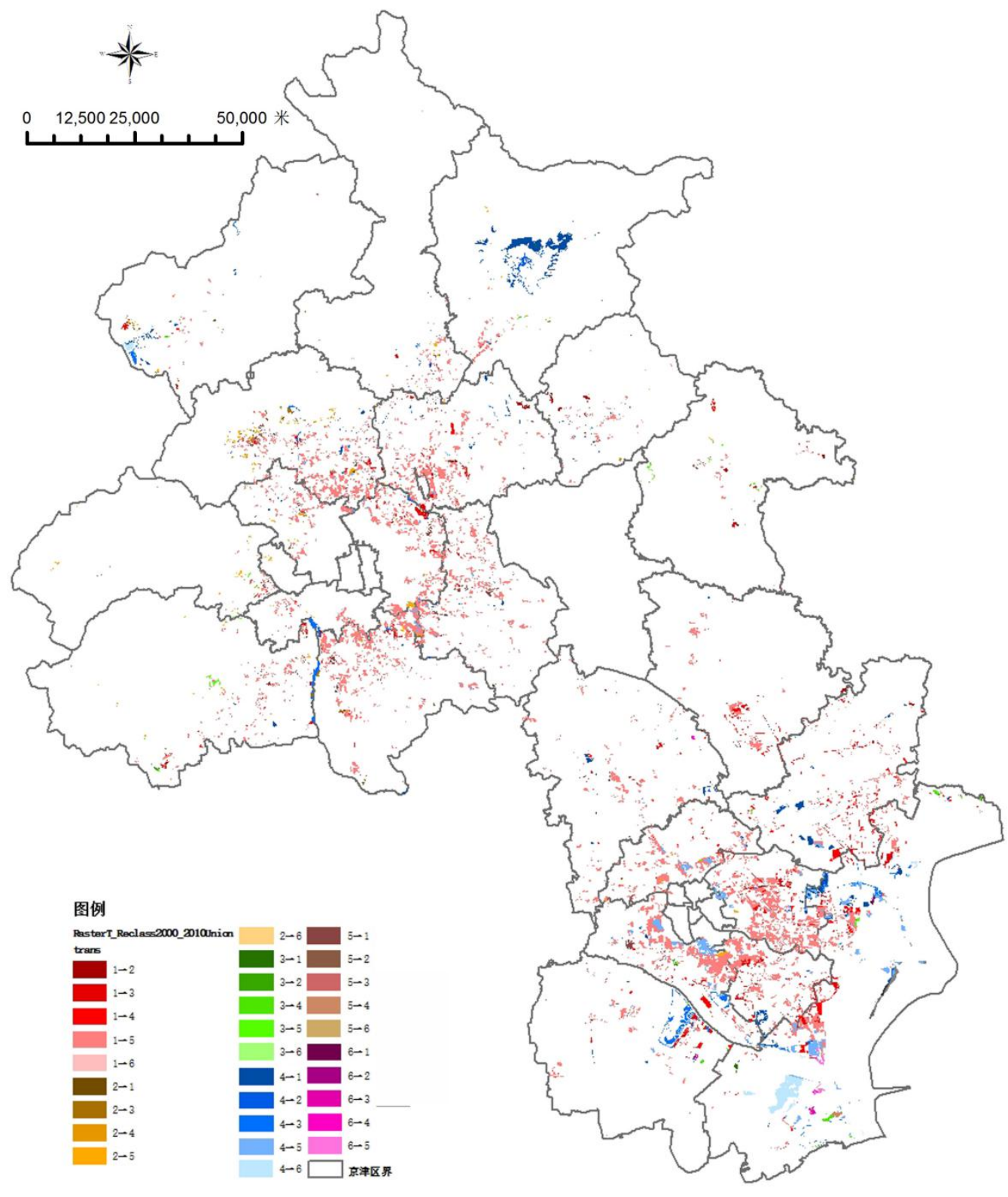

Figure 3.8 Distribution of landscape type transfer in 2000-2010(In the legend, "1", "2", "3", "4", "5" and "6" respectively represent cultivated land landscape, woodland landscape, grassland landscape, water landscape, construction land landscape and unused land landscape.)Source: Photo by author. 


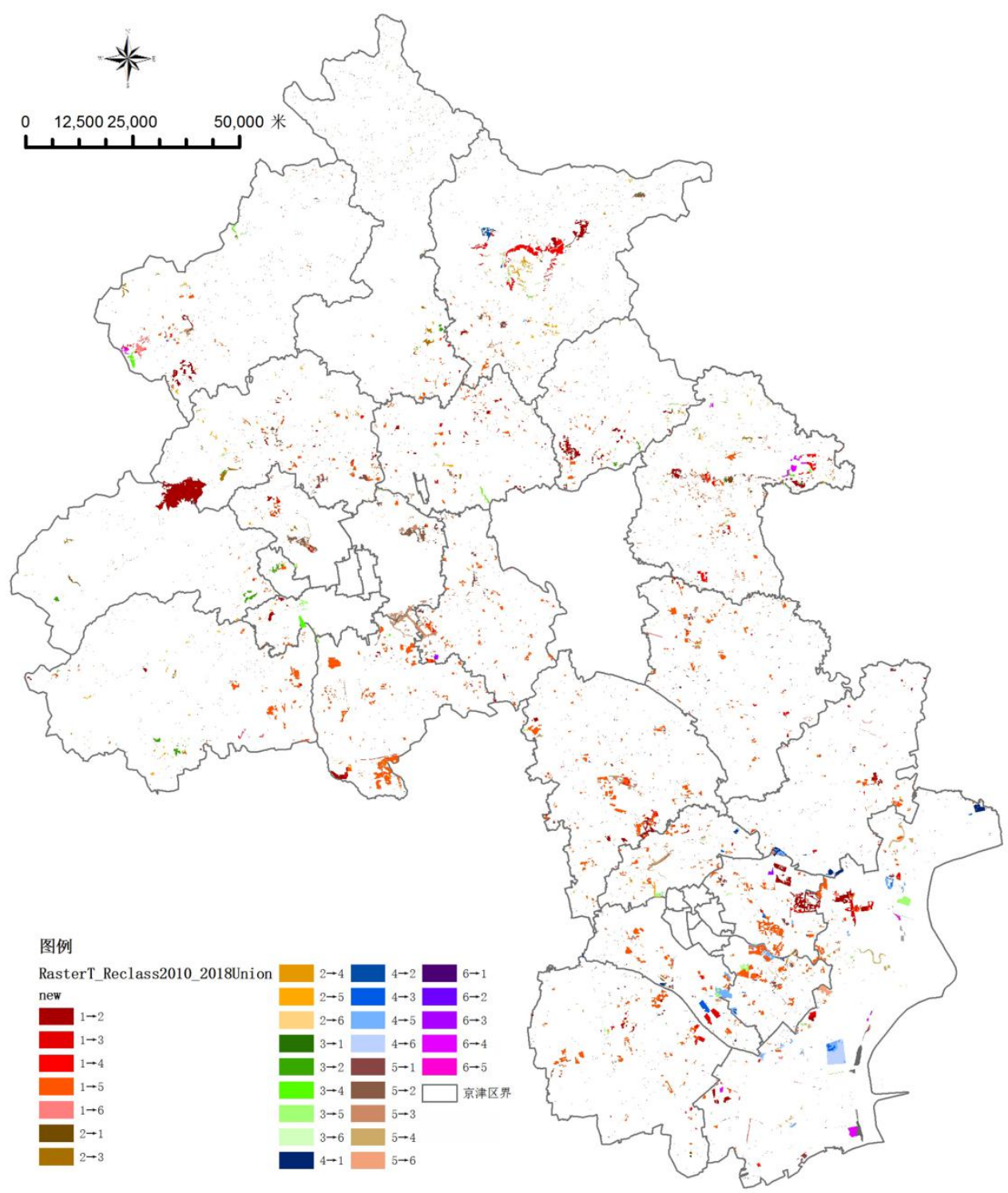

Figure 3.9 Distribution of landscape type transfer in 2010-2018(In the legend, "1", "2", "3", "4", "5" and "6" respectively represent cultivated land landscape, woodland landscape, grassland landscape, water landscape, construction land landscape and unused land landscape.)Source: Photo by author.

\section{Conclusions}

The spatial differentiation of rural natural landscape in Beijing and Tianjin is significant, forming a natural landscape pattern of "mountain, field and sea", which has four spatial characteristics of scale, distribution, network and shape. However, the high spatial differentiation of rural natural landscape types is not balanced on the whole, leading to the simplicity of rural surrounding natural landscape types, and the rural landscape features in Beijing and Tianjin are not obvious.

In the process of rapid urbanization, the change process of rural natural landscape elements in Beijing and Tianjin is mainly the process of farmland landscape transferring to construction land landscape. The construction land is centered on the urban center and rural residential area, and expands to the periphery in a circular manner, while the cultivated land landscape shrinks and presents a fragmented structure. 
In the process of rapid urbanization, the pattern of rural natural landscape elements in Beijing and Tianjin has a process of decreasing in scale, increasing in fragmentation degree and continuous fluctuation in shape. The pattern of rural natural landscape in Beijing and Tianjin tends to be fragmented, discrete and heterogeneous, and its shape changes from complex to regular. The transformation, dispersion and integration of rural natural landscape patches in Beijing and Tianjin are passive, and there is no effective ecological restoration and scientific planning after being destroyed.

\section{References}

[1] YU,T., YUAN,Q., LENG,H..'Research on the Evaluation of Rural Landscape Vulnerability in County Villages: A Study Case of Harbin County Villages', Chinese Landscape Architecture, 2019,35(11):87-91.

[2] Yuan, Q., Ma, Y., Leng, H.. 'A Study on Establishing of Rural Landscape Style Along the Gui River Based on Ecological Prospect', Urbanism and Architecture. 2017(21): 113-117.

[3] Zhang, L., Wang, L., Li, R.. 'Discussions on the Dilemmas, Causes and Conservation Suggestions of Rural Landscape in China Based on the Field Studies', Urban Planning International. 2019, 34(05): 59-68.

[4] XU,X., LIU, J., ZHANG, S., et al. 'China multi-period land use and land cover remote sensing monitoring data set (CNLUCC) . Registration and Publication System of Data Center for Resources and Environmental Sciences', Chinese Academy of Sciences, 2018. DOI: 10.12078/2018070201.

[5] V.Whitford, A.R.Ennos, J.F.Handley. 'City form and natural process--indicators for the ecological performance of urban areas and their application to Merseyside, UK'. Landscape and Urban Planning. 2001(57):91-03.

[6] Foltête J, Girardet X, Clauzel C. 'A methodological framework for the use of landscape graphs in landuse planning'. Landscape and Urban Planning. 2014, 124: 140-150.

[7] Guo, L., Di, L., Tian, Q.. 'Detecting spatio-temporal changes of arable land and construction land in the Beijing-Tianjin corridor during 2000-2015'. Journal of Geographical Sciences. 2019, 29(5): 702-718.

[8] Zhen, F., Qin, X., Ye, X., et al. 'Analyzing urban development patterns based on the flow analysis method'. Cities. 2019, 86: 178-197.

[9] Marco,V., Maurizia, S. 'Landscape sequences along the urban-rural-natural gradient: A novel geospatial approach for identification and analysis'. Landscape and Urban Planning. 2005(8):42-45.

[10] Innocent, C. 'Opportunities in master and local planning for resilient rural settlement in Zimbabwe'. Journal of Rural Studies. 2021(8):97-105.

[11] Jizhe, Z., Quanhua, H. 'Resilience assessment and planning of suburban rural settlements based on complex network'. Sustainable Production and Consumption. 2021(10):1645-1662.

[12] Tom, M. 'Planning for place: Place attachment and the founding of rural community land trusts'. Journal of Rural Studies. 2021(4):21-29.

[13] Camille, L., Romain, M.. 'Scheduling urbanization in rural municipalities: Local practices in land-use planning on the fringes of the Paris region'. Land use policy. 2020(12):105040.

[14] Setu, P. ‘Disaggregated household energy supply measurement to support equitable municipal energy planning in rural Nepal'. Energy for Sustainable Development. 2020(12):8-21.

[15] Lei, X., Wen, C. 'Sustainable development strategy of rural built-up landscapes in Northeast China based on ANP approach'. Energy procedia. 2019(1):844-850.

[16] Fang, F., Libang, M., Hao, F., et al. 'The spatial differentiation of quality of rural life based on natural controlling factors: A case study of Gansu Province, China'. Journal of Environmental Management. 2020(6):110439.

[17] Fabrizio, A., Paola, G.,Marco, D., et al. 'Towards reconstructing rural landscapes: A case study of Italian Mongardino'.Journal of Rural Studies. 2021(7). 
$\mathrm{Xu}, \mathbf{N}$.

[18] Jayme E, W. 'More than meets the eye: Organizational capacity of nonprofits in the poor, rural South'. Journal of Rural Studies. 2021(8):497-507.

[19] Akhilesh, N., Sunil, S. 'Condition Based Maintenance Planning of low volume rural roads using GIS'. Journal of Cleaner Production. 2021(8):127649.

[20] Jessica, W. 'Making the countryside more like the countryside? Rural planning and metropolitan visions in post-quake Chengdu'. Geoforum. 2017(1):110-118. 